\title{
Técnicas de medida para la capacitancia y resistencia eléctrica de una membrana de bicapa lipídica
}

Technical measure for capacitance and resistance of a lipid bilayer membrane

\author{
CARLOS RAMÍREZ-MARTÍN \\ Físico \\ MSc. Metalurgia y Ciencia de los Materiales \\ Grupo de Investigación GIMAC \\ Universidad de Boyacá, Colombia \\ cramirezm@uniboyaca.edu.co \\ ÓSCAR DURÁN-AVENDAÑO \\ Físico \\ MSc. Instrumentación Física \\ oscar.duran@uptc.edu.co
}

Universidad de Pedagógica y Tecnológica de Colombia, Colombia 


\title{
RESUMEN
}

Las membranas de bicapa lipídica plana (BLM) han sido utilizadas en un variado número de aplicaciones que van desde la biofísica, la investigación práctica, el estudio de microchips y su desarrollo como biosensores utilizando membranas de bicapa lipídicas soportadas. Se presenta una visión general sobre el origen del concepto de bicapa lipídica, su asociación con el comportamiento de un circuito eléctrico RC en paralelo y sus propiedades eléctricas más relevantes, capacitancia y resistencia eléctrica. Se exploran las técnicas instrumentales utilizadas para cuantificar estas propiedades eléctricas y que permiten caracterizar el desempeño de BLM frente a su interacción con una diversidad de agentes fisicoquímicos externos.

Palabras clave: Membrana de bicapa lipídica (BLM), resistencia eléctrica, capacitancia, fosfatidilcolina.

\begin{abstract}
The planar lipid bilayer membranes (BLM) have been utilized in a number of different applications like biophysics, practical research, study of microchips and their development as biosensors using supported bilayer lipid membranes. An overview on the origin of the concept of lipid bilayer, its association with the behavior of a parallel circuit RC and its most important electrical properties, capacitance and electrical resistance is presented. The instrumental techniques used to quantify these electrical properties that allow characterize the performance of BLM against its interaction with diversity of external physical-chemical agents, are explored.
\end{abstract}

Keywords: Bilayer lipid membrane (BLM), electrical resistance, capacitance, phosphatidylcholine. 


\section{INTRODUCCIÓN}

Los primeros estudios sobre la composición básica de las membranas celulares se iniciaron con la observación de las tasas de acumulación intracelular de sustancias y su proporcionalidad con la solubilidad en los lípidos (Overton, 1889). Danielli y Davson (1935), produjeron un modelo de la membrana de la célula en la que una capa lipoidal se intercala entre dos capas proteínicas. La primera medición directa del espesor obtenido a partir de electromiografías por Robertson (1959), sugirió que la capa lipoide era probablemente sólo tan gruesa como dos moléculas comunes de lípidos biológicos.

Usando mediciones de impedancia eléctrica Fricke \& Morse (1925) proporcionaron la primera estimación del espesor de las membranas (2 $\mathrm{nm}$ a $3 \mathrm{~nm}$ ), a partir de las mediciones de la impedancia eléctrica de células en suspensión. El concepto de la "unidad de membrana" se cerró cuando Mueller, Rudin, Tien y Wescott (1962) produjeron la primera membrana de bicapa lipídica plana (BLM) in vitro a partir de lípidos extraídos de cerebro bovino. Esta situación generó muchos otros estudios y las bicapas lipídicas se formaron a partir de una amplia variedad de lípidos extraídos de tejidos biológicos, así como de anfófilos sintéticos (Mueller et al., 1962; Mueller et al., 1964; Tien et al., 1968; Tien y Dianna 1967; Tien 1975; Goldup, 1970) y un estudio reciente realizado por (Tien \& Ottova, 2001).

La caracterización de las BLM desde el punto de vista eléctrico y su asociación con un circuito eléctrico RC en paralelo, ha llevado a profundizar en el comportamiento de estas unidades estructurales frente a diferentes agentes externos que entran en contacto. Por tal motivo conocer las variaciones de la capacitancia y la resistencia eléctrica permitirán dar respuesta a diferentes inquietudes de orden fisiológico y farmacológico. En este trabajo se presenta una revisión sobre la evolución del concepto de unidad estructural de la membrana y sus propiedades eléctricas, al igual que se incluye una exploración de las técnicas instrumentales más utilizadas que permiten cuantificar la capacitancia y la resistencia eléctricas de las BLM.

\section{ESTRUCTURA DE LA MEMBRANA}

La membrana biológica es uno de los componentes estructurales más importantes y funcionales de la célula; cumple con una serie de funciones importantes de orden: estructural, de contacto, de recepción y de transporte (Sim, 1982; Alberts et al., 1998; Cevc \& Marsh 1987; Jain, 1988). La estructura de la biomembrana tiene varias características comunes como se observa en la Figura 1; su base es una bicapa lipídica compuesta de moléculas de lípidos en los cuales se incorporan proteínas integrales y periféricas. Las proteínas son apoyadas o cubiertas por proteínas estructurales, como por ejemplo la 
espectrina neta en los eritrocitos (Alberts et al., 1998) o la proteína S bacteriana (Pum y Sleytr, 1999). La parte exterior de las membranas bacterianas y vegetales también está cubierta por polisacáridos (Alberts et al., 1998). La matriz de lípidos proporciona la integridad de la membrana, el aislamiento eléctrico y las proteínas determinan el cumplimiento de las funciones específicas de la membrana.

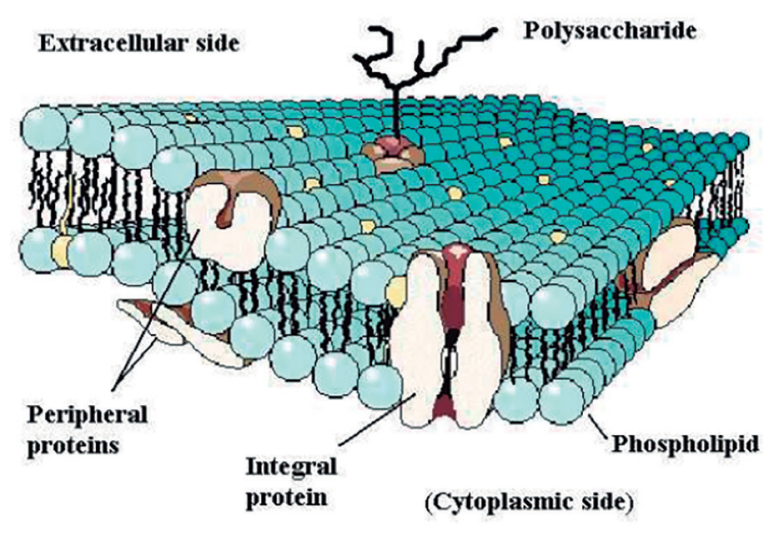

Figura 1. Modelo estructural de una biomembrana.

Fuente: Hianik, 2006.

En condiciones fisiológicas normales, la bicapa lipídica se encuentra en estado cristalino-líquido; desde el punto de vista físico la biomembrana representan un cristal líquido de tipo esméctico (Nagle \& Tristram-Nagle, 2000). El espesor de las biomembranas varía entre $5 \mathrm{~nm}$ a $10 \mathrm{~nm}$ y es considerablemente menor que la dimensión de las células. Las variaciones de espesor de una biomembrana se deben principalmente a las proteínas integral, periférica y estructural, así como a la presencia de lipopolisacáridos y glycopolysacaridos (Alberts et al., 1998).

El progreso en la comprensión de las particularidades de la estructura y las propiedades de la membrana, se conecta directamente con el progreso de la física. La aparición del microscopio y el considerable progreso en la fabricación de lentes ópticas en el siglo XVII permitió realizar la primera observación de la estructura celular por Hooke en 1662. Sin embargo, el mayor avance era muy lento y sólo en 1831, Brown mostró la existencia de un núcleo en la célula (Roland, 1974). Entonces la teoría celular, uno de los conceptos fundamentales de la biología, fue formulada por el botánico Schleiden y el zoólogo Schwann (Alberts et al., 1998). La existencia de la membrana que rodea la célula fue propuesta en 1855 por Negeli, quien observó que células en buen estado puede cambiar el volumen con los cambios de la presión osmótica del ambiente que la rodea. Estos experimentos fueron continuados por Overton (Sim, 1982), quien demostró que moléculas no polares penetran más fácil a través de la membrana celular, que las moléculas polares. Los resultados de estos experimentos le permitieron plantear la hipótesis de que la estructura de la membrana tiene una naturaleza lipídica. 
Un mayor desarrollo del concepto de la estructura de la membrana se logró gracias al trabajo de Gortel y Grendel (1925), en el primer tercio del siglo XX. Esta época se caracterizó por un excelente avance en el estudio de las capas monomoleculares en la interfaz aire-agua que es debida al trabajo de Langmuir y compañeros de trabajo (Langmuir, 1933; Langmuir \& Waugh, 1938). Se extrajeron lípidos a partir de eritrocitos y se mostró que el área de la capa monomolecular formada por los lípidos en una interfaz aire-agua es el doble de la superficie de las células de eritrocitos. Esto dio como resultado el concepto sobre la composición de la biomembrana con dos capas de lípidos monomoleculares como se observa en la Figura 2a.

A pesar de ciertos errores en su estudio, fundamentados en una subestimación de la concentración de los lípidos y del área de los eritrocitos, así como no tener en cuenta la presencia de proteínas, el concepto de la estructura de bicapa de la biomembrana fue aceptado por gran parte de la comunidad científica. Actualmente está claro que si se fuera a realizar el experimento correctamente, la interpretación sería diferente. Se sabe ahora que la membrana del eritrocito está compuesta por un $43 \%$ de lípidos, el $49 \%$ de proteínas y un $8 \%$ de hidrocarburos.

La suposición de la conexión de las proteínas con las membranas fue planteada 10 años más tarde por Danielli y compañeros de trabajo (Danielli y Dawson, 1935), debido a la necesidad de explicar sustancialmente la baja tensión superficial de las biomembranas, en comparación con monocapas de lípidos puros en la interfaz aire-agua. Por ejemplo, la tensión superficial de la membrana de la célula de erizo de mar es de aproximadamente $0.2 \mathrm{mNm}^{-1}$, mientras que para las monocapas de ácidos grasos en la interfaz aire-agua se encuentra entre $10 \mathrm{mNm}^{-1} \mathrm{a} 15 \mathrm{mNm}^{-1}$ (De Robertis et al., 1970).

Otros estudios mostraron que la adición de las proteínas en la subfase de agua causan una disminución en la tensión superficial de las monocapas de lípidos, por tanto se ha propuesto que las proteínas globulares están conectados con ambas superficies de la bicapa lipídica como se aprecia en la Figura 2b. La comprobación directa de la existencia de la biomembrana ha sido posible al descubrimiento del microscopio electrónico y su aplicación en la biología. La primera micrografía realizada con un microscopio electrónico, mostró que la célula está rodeada por una delgada membrana con un espesor de aproximadamente $610 \mathrm{~nm}$. Esta membrana está compuesta por tres capas; dos capas de alta densidad electrónica con un espesor de aproximadamente $2 \mathrm{~nm}$ separadas por una capa de baja densidad electrónica con un espesor de $3.5 \mathrm{~nm}$ (Zetterquist, 1956). Se ha propuesto que las capas de electrones de alta densidad corresponden a la región de grupos de cabeza polares de la capa de fosfolípidos cubiertos por las proteínas, mientras que la capa de baja densidad electrónica corresponde a la parte hidrófoba de la bicapa lipídica.

De acuerdo con este modelo, la bicapa lipídica está cubierta por una capa de proteínas en una conformación $\beta$, que son adyacentes a la parte polar de la membrana debida a interacciones electrostáticas. La asimetría se debe al hecho de que la monocapa exterior está cubierta por glicoproteínas, según 
se observa en la Figura 2c. En la figura 2d se muestra el modelo de membrana compuesto de micelas cubiertas por proteínas, propuesto por Lucy (De Robertis et al., 1970). Sin embargo, este modelo no puede explicar la pequeña conductividad de las BLM determinado por (Mueller et al., 1962), este y estudios posteriores demostraron que la conductancia específica de las BLM está comprendida en el rango de $10^{-6} \Omega^{-1} \mathrm{~cm}^{-1}$ a $10^{-10} \Omega^{-1} \mathrm{~cm}^{-1} \quad$ (Tien, 1974; Ivkov \& Berestovsky, 1981).

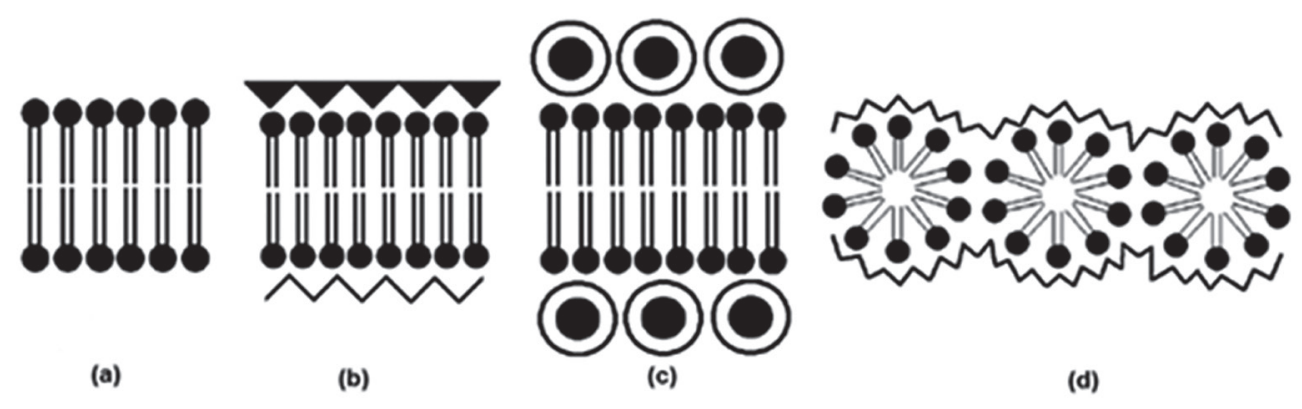

Figura 2. Resumen histórico esquemático de los modelos de biomembranas. a) Bicapa lipídica de Gortel y Grendel. Fuente: Gortel 8 Grendel, 1925. b) Modelo de Danielli y Dawson. Fuente: Danielli \& Dawson, 1935. c) Modelo unitario de biomebrana por Robetson. Fuente: Zetterquist, 1956. d) Modelo micelar propuesto por Lucy.

Fuente: Robetson, 1959.

La alta resolución de la microscopía electrónica, así como los métodos mejorados para la preparación de muestras ultra delgadas permitió obtener micrografías de las membranas celulares con detalles estructurales adicionales. El análisis de estos resultados permitió proponer el llamado modelo del mosaico fluido de biomembranas (Singer \& Nicolson, 1972; Finean et al., 1978). Según este modelo representado en la Figura 1, la membrana está compuesta por una bicapa de lípidos con proteínas incorporadas. El modelo permitió explicar la dependencia de la actividad de proteínas de membrana en el estado físico de la membrana, así como la existencia de la viscosidad de la membrana.

Actualmente varias modificaciones al modelo del mosaico fluido se utilizan para la descripción de la estructura de las biomembranas. Sin embargo, estudios de las propiedades físicas de las biomembranas y bicapas lipídicas revelaron que la movilidad de algunas proteínas de la membrana está fuertemente restringida (Danielli, \& Dawson, 1935; Rogers et al., 1980). Estudios teóricos y experimentales realizados recientemente han demostrado que la estructura lateral de la bicapa lipídica es dinámica, heterogénea y se caracteriza por la formación de dominios de lípidos con diferente movilidad de los lípidos y de las proteínas (Sim, 1982 \& Alberts et al., 1998) 


\section{PROPIEDADES ELÉCTRICAS}

Las bicapas lipídicas tienen una capa central, no polar, que es de unos $2 \mathrm{~nm}$ a $3 \mathrm{~nm}$ de espesor, que es determinada por la energía de Born requerida para mover un ión del medio acuoso donde la constante dieléctrica es $\varepsilon_{\widetilde{\tau}} 78$ al interior de la bicapa (Tien, 1966; Fettiplace et al., 1971; Coster \& Smith, 1974; Hanai et al., 1964, Huang \& Levitt 1977). La energía de Born para las particiones al interior de la bicapa lipídica de un medio acuoso está dada por la ecuación 1(Neumcke \& Läuger, 1969).

$E_{B}=\frac{z^{2} e^{2}}{8 \pi \varepsilon_{0} R}\left[\frac{1}{\varepsilon_{m}}-\frac{1}{\varepsilon_{w}}\right]$

Donde:

$R$ es el radio del ion; $e$ es la carga del electrón; $\mathrm{z}$ es la valencia del ion; $\varepsilon_{\mathrm{m}}$ es la constante dieléctrica del interior de la membrana; $\varepsilon_{\mathrm{w}}$ es la constante dieléctrica del agua y $\varepsilon_{0}$ es la permitividad del espacio.

Para un ion Potasio, por ejemplo, esta energía de partición de Born (Coster, 1999) es del orden de 3 $\mathrm{eV}$. La conductancia eléctrica de la BLM es muy baja ya que las concentraciones de iones en la membrana son extremadamente bajas, incluso si las movilidades de los iones en la membrana fueran altas. La baja conductividad de este fluido flexible y la estructura de membrana, es de importancia en las propiedades funcionales de la membrana celular. La baja conductancia de las membranas lipídicas son, sin embargo, varias decenas de órdenes de magnitud mayor que el esperado a partir de la concentración de portadores en el lípido de la membrana; la explicación de ello radica en la formación de los "defectos" de poro.

\section{Circuito equivalente de membrana}

Fricke, \& Morse (1925), llevaron a cabo el siguiente experimento; dispusieron de glóbulos rojos (RBC) que se suspendieron en una solución salina isotónica y a continuación dos electrodos de Pt se colocaron en la suspensión y se conectaron a un brazo de un puente de Wheatstone; la conductividad y la capacitancia de la suspensión RBC, se midió como función de la frecuencia.

A bajas frecuencias la impedancia de la suspensión de RBC es muy alta, mientras que a altas frecuencias la impedancia disminuye a un valor bajo. Para explicar los hallazgos, Fricke propuso un modelo donde los RBC estaban rodeados por una delgada capa de material dieléctrico, eléctricamente equivalente a una resistencia $\left(R_{m}\right)$ y un condensador $\left(C_{m}\right)$ en paralelo. El circuito eléctrico que se ajusta a las condiciones anteriormente descritas se representa en la Figura 3 y se conoce como circuito equivalente de membrana. Así, las líneas de corriente fluían alrededor de los glóbulos rojos a bajas 
frecuencias. A muy altas frecuencias, la resistencia se vuelve muy baja debido a que toda la corriente se desvía a través del condensador.

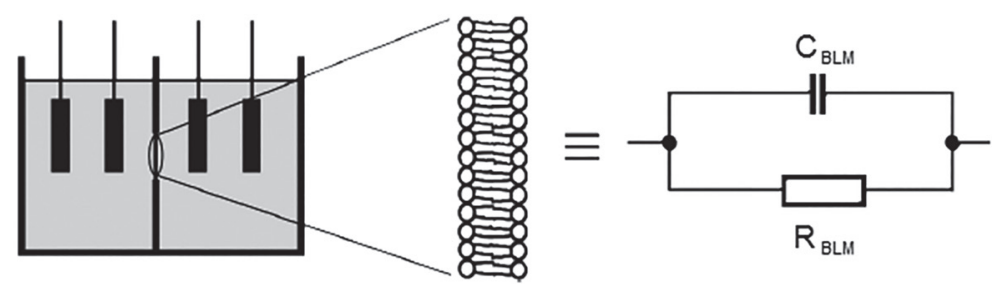

Figura 3. Circuito equivalente de BLM.

Fuente: Kramack, et al., 2007

Hober (1910), encontró que las suspensiones de glóbulos rojos tienen una alta resistencia eléctrica, mientras que el citoplasma tiene una conductividad similar a la solución fisiológica salina. A partir de este hecho, Hober concluyó que la membrana celular tiene una resistencia eléctrica alta. Utilizando la fórmula para un condensador de placas paralelas, Fricke determinó que la capacitancia $\left(\mathrm{C}_{\mathrm{m}}\right)$ de los glóbulos rojos era de $0.81 \mathrm{mFcm}^{-2}$. Para un condensador de placas paralelas, la capacitancia está descrita por la ecuación 2

$C_{m}=\frac{\varepsilon}{4 \pi t_{m}}$

\section{Donde:}

$\mathrm{C}_{\mathrm{m}}$ es la capacitancia de la membrana, $\varepsilon$ la constante dieléctrica de la membrana y $\mathrm{t}_{\mathrm{m}}$ el espesor de la membrana.

Fricke determinó que el espesor de la membrana de los glóbulos rojos era de $3.3 \mathrm{~nm}$ y $11 \mathrm{~nm}$, asumiendo $\varepsilon=3$ y $\varepsilon=10$, respectivamente (Fricke \& Morse 1925). De hecho, modernas mediciones en membranas de bicapa de lípidos artificiales y biomembranas confirman plenamente la estimación de Fricke. 


\section{MÉTODOS PARA DETERMINAR LA RESISTENCIA Y LA CAPACITANCIA DE LAS BLM CAPACITANCIA}

La capacitancia, es el parámetro considerado la mejor herramienta para determinar la estabilidad y la integridad de las BLM y por esta razón se mide para todas las bicapas, incluso cuando otras propiedades son el centro principal de las mediciones. Existen tres métodos principales para la determinación de la capacitancia de la bicapa lipídica plana: método de la descarga, método de conversión de la capacitancia a período y método de conversión de capacitancia a voltaje. Para la comparación entre diferentes estudios, los valores medidos de la capacitancia deben ser normalizados al tamaño de la superficie, la capacitancia específica de la bicapa lipídica plana, es decir, la capacitancia por unidad de área.

\section{Método de la descarga}

El método más simple y común para la medición de la capacitancia de una bicapa lipídica plana es la medición de la constante de tiempo del voltaje de descarga (Troiano et al 1988, Benz \& Janko 1976, Kramar et al 2007, Wilhelm et al 1993, Diederich et al 1998, Meier et al 2000, Sharma et al 1996, Kramar,et al 2009, Vargas et al 2000). Para su implementación se necesitan un generador de voltaje, un interruptor y un osciloscopio. Para realizar la medición, una bicapa lipídica plana se carga primero por un pulso de voltaje; al final del pulso, la bicapa de lípido cargada se descarga a través de una resistencia conocida y el proceso de descarga se monitorea con un osciloscopio, presentando un comportamiento como el observado en la Figura 4.

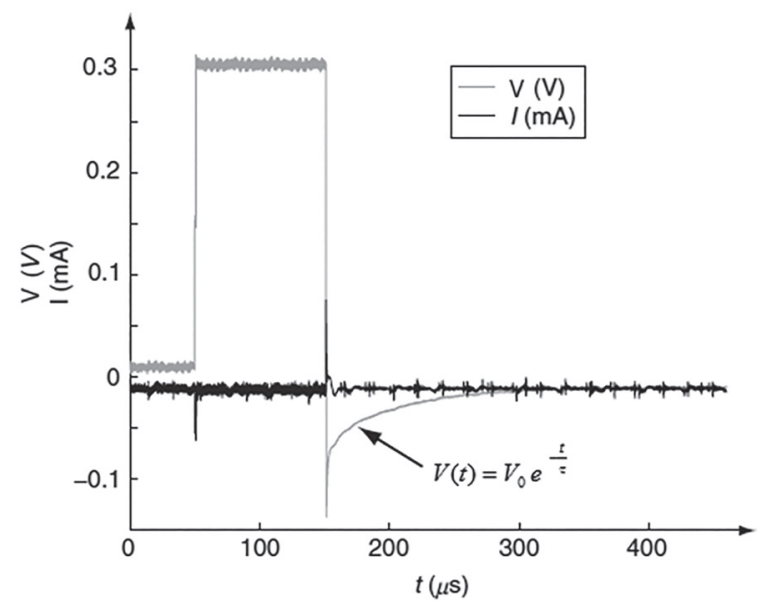

Figura 4. Medida de la capacitancia de BLM por el método de la descarga Fuente: Kramack, et al., 2010. 
El voltaje $\mathrm{V}_{(\mathrm{t})}$ en la bicapa lipídica plana disminuye exponencialmente y es descrito a través de la ecuación 3

$$
V(t)=V_{0} e^{-\frac{t}{\tau}}
$$

Donde:

$\mathrm{V}_{0}$ es la amplitud del pulso y $\mathrm{t}$ es la constante de tiempo.

La constante de tiempo descrita por la ecuación 4, depende de la capacitancia C y la resistencia $\mathrm{R}$ que proceden de la bicapa lipídica plana y el circuito electrónico

$\tau=R C$

La resistencia del circuito electrónico es generalmente conocida y es muy inferior a la resistencia de la bicapa lipídica plana $\approx 10^{8} \Omega$, por lo tanto, la capacitancia de la bicapa lipídica plana se puede determinar en dos etapas. En primer lugar, la capacitancia del sistema electrónico $C_{S Y S}$ se mide sin la bicapa lipídica plana. A continuación, se determina la capacitancia del sistema electrónico con la bicapa lipídica plana y una solución salina $\mathrm{C}_{\mathrm{SLBM}}$. La capacitancia de la bicapa lipídica plana $\mathrm{C}_{\mathrm{LBM}}$ se obtiene como la diferencia entre $\mathrm{C}_{\text {SYS }} \mathrm{y}_{\text {SLBM }}$ expresada por la ecuación 5

$$
C_{B L M}=C_{S Y S}-C_{S B L M}
$$

\section{Método de conversión de capacitancia a periodo}

Los parámetros eléctricos de la bicapa lipídica plana también se puede medir por medio de una señal de voltaje alterno, que ofrece la ventaja de eliminar el posible efecto del electrodo de polarización. Kalinowski \& Figaszewski (1992) construyeron el instrumento esquematizado en la Figura 5, que convierte la capacitancia de una bicapa lipídica plana en un tren de pulsos rectangulares. Durante la medición, la bicapa lipídica plana se carga y se descarga con una corriente constante. La duración del ciclo de carga-descarga es proporcional a la capacitancia de la membrana. En el circuito de la Figura 5 , la capacitancia de la bicapa lipídica plana es representada por el condensador $\mathrm{C}_{\mathrm{LBM}}$. 


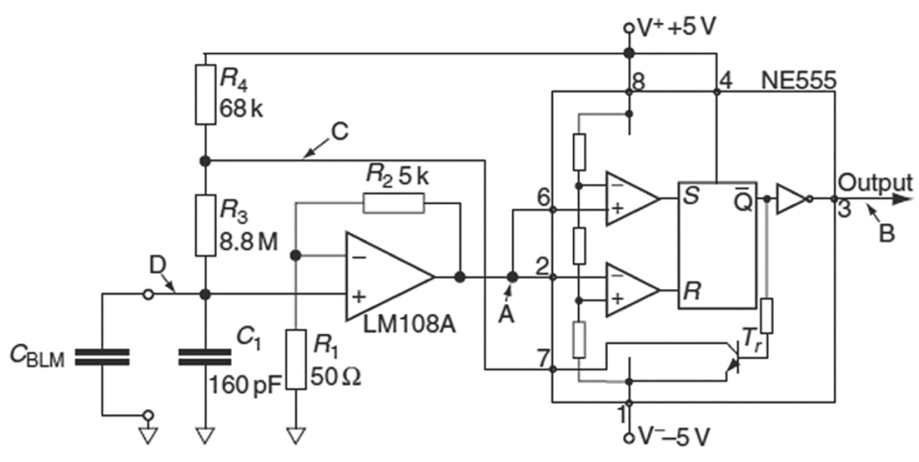

Figura 5. Diagrama esquemático de conversión de capacitancia a periodo Fuente: Kalinowski, \& Figaszewski, 1992.

El voltaje en el punto D se amplifica con un amplificador no inversor de ganancia $\mathrm{K}$ descrita por la ecuación 6

$\kappa=\frac{R_{1}+R_{2}}{R_{2}}$

La señal amplificada es una de las entradas del circuito integrado NE555. Dos comparadores de voltaje se encuentran en el circuito integrado, ambos con una de sus entradas conectadas al voltaje de la salida del amplificador (A) mientras la otra entrada está conectada al voltaje del divisor de voltaje, el cual es realizado por tres resistencias. Dependiendo de los niveles de voltaje en las entradas R y S, la salida (B) del circuito cambia de un estado bajo a un estado alto. El resultado es una señal de onda cuadrada, que tiene un período bien definido. A través de la Ecuación 7 se puede calcular el voltaje a través de la bicapa lipídica plana

$V_{B L M}=\frac{2}{3} V \frac{1}{\kappa}=\frac{2 V R_{1}}{3\left(R_{1}+R_{2}\right)}$

La medida se divide en dos pasos. En primer lugar, el condensador está cargado con la corriente que pasa a través de las resistencias $R_{4}, R_{3}$ y el transistor $T$ está apagado. A continuación, el condensador se descarga debido a la corriente a través del transistor, que es una consecuencia de un cambio de estado del flip-flop. La corriente fluye a través de la resistencia $R_{3}$ y el transistor $T_{r}$. La Ecuación 8 , permite calcular el producto del voltaje y el tiempo en un período

$T V=V_{B L M}\left(R_{3}+R_{4}\right) C_{B L M}+V_{B L M} R_{3} C_{B L M}$ 
Si $R_{3} \ll R_{4}$ y $V \quad V \quad \mid V$ y entonces el periodo se determina con la ecuación 9

$T=\frac{2 V_{B L M} R_{3} C_{B L M}}{V}$

\section{Método de conversión de capacitancia a voltaje}

Cuando una señal alterna es aplicada a una bicapa lipídica plana, su impedancia eléctrica cobra importancia. Dado que los condensadores conducen corriente en proporción a la tasa de cambio del voltaje, ellos pasan más corriente para cambios rápidos de voltaje, y menos corriente para cambios lentos de voltajes. Por lo tanto, la parte capacitiva de la impedancia-capacitiva para cualquier condensador es inversamente proporcional a la frecuencia de la corriente alterna. De acuerdo con esta teoría, la capacitancia de bicapa lipídica plana se mide utilizando un puente de Wheatstone de corriente alterna como lo muestra la Figura 6, (Hanai et al 1964; Rosen \& Sutton 1968; White 1970), que contiene una resistencia variable en paralelo con un condensador variable en la rama conocida. Cuando el puente está equilibrado a una frecuencia dada, la configuración de la rama conocida suministra la capacitancia equivalente en paralelo y la resistencia del circuito es conectada a los terminales desconocidos. Dado que la bicapa lipídica plana se sumerge en el electrolito, el puente de Wheatstone mide la impedancia equivalente en paralelo del sistema membrana-electrolito.

La capacitancia paralelo equivalente puede ser representada por la capacitancia de la membrana, la capacitancia parásita asociada a los electrodos en serie con la resistencia del electrolito, por lo tanto, el circuito equivalente apropiado y la transformación de ecuaciones debe ser usada para relacionar la capacitancia de la membrana con los elementos del puente de Wheatstone. 


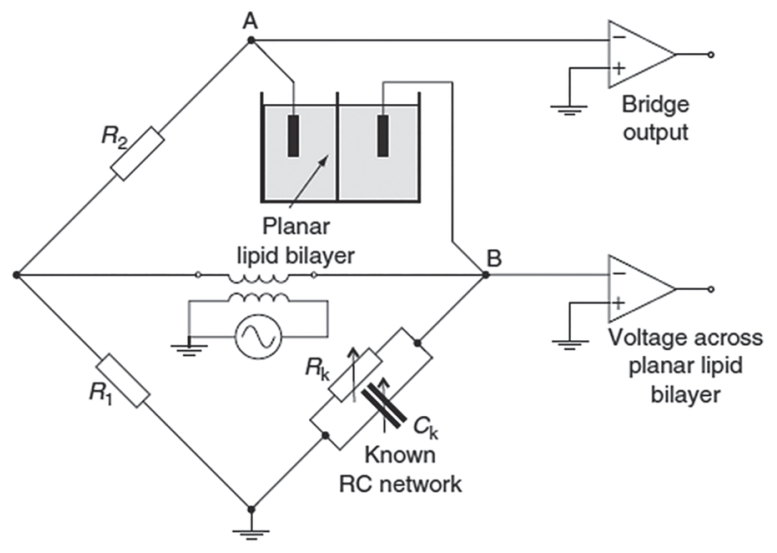

Figura 6. Diagrama esquemático de conversión de capacitancia a voltaje Fuente: Hanai et al 1964; Rosen \& Sutton 1968; White 1970

Micelli et al., (2000) midieron la capacitancia de bicapas lipídicas planas con la aplicación de un voltaje alterno sinusoidal con una amplitud de $2 \mathrm{mV}$ y una frecuencia de $1 \mathrm{kHz}$. A esta alta frecuencia, casi todos los cruces de corriente de la parte reactiva de las bicapas lipídicas planas y su resistencia son despreciables. El voltaje rectificado es proporcional a la capacitancia de la bicapa lipídica plana. Mediante el uso de un conjunto de valores de prueba para la capacitancia, que fueron incluidos uno a uno en el sistema de medición, parametrizaron la relación entre voltaje medido y capacitancia y obtuvieron una relación hiperbólica descrita por la ecuación 10, con dos parámetros conocidos $a$ y $b$ :

$$
C_{B L M}=a \frac{V_{1 h}}{b-V_{1 h}}
$$

\section{RESISTENCIA}

Las membranas de bicapa lipídica plana presentan una resistencia en el intervalo de pocos giga ohm. La resistencia se calcula generalmente de acuerdo con la ley de Ohm como una relación de voltaje aplicado a la bicapa lipídica plana y la corriente que fluye a través de esta. Como se mencionó anteriormente, los parámetros eléctricos de la bicapa lipídica plana también se puede medir por medio de una corriente alterna. El continuo control de la capacitancia es útil para conocer el espesor de la membrana, mientras que el monitoreo continuo de la resistencia permite estudiar las interacciones proteína-lípido y las fluctuaciones de la bicapa lipídica plana. 
Gallucci et al., (1996) presentaron un circuito eléctrico adecuado para el monitoreo continuo de la capacitancia y resistencia de la bicapa lipídica plana simultáneamente. Un voltaje de entrada se compone de dos señales que varían sinusoidalmente: uno con amplitud variable de $0.1 \mathrm{~V}$ a $1.5 \mathrm{~V}$ y con frecuencia de $1 \mathrm{~Hz}$ y otro con una amplitud de $2 \mathrm{mV}$ y frecuencia de $1 \mathrm{kHz}$. La bicapa lipídica plana y el dispositivo de medición se muestran en los circuitos equivalentes del lado izquierdo de la Figura 7.

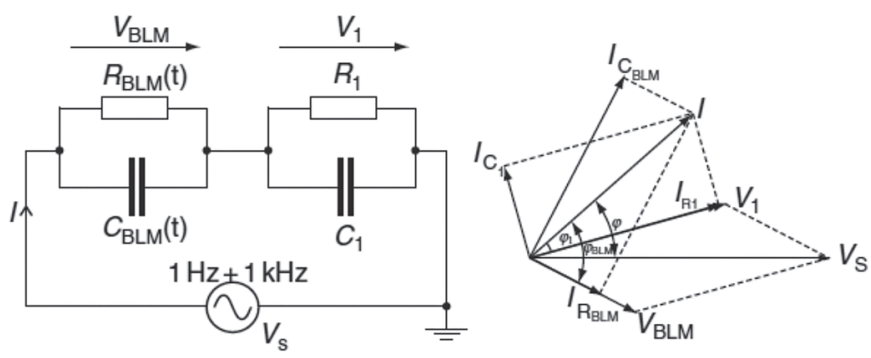

Figura 7. Izquierda: Circuito equivalente de BLM y piezas de medida. Derecha: esquema vectorial de voltajes y corrientes. Fuente: Gallucci et al., 1996; Micelli, et al., 2000 \& Micelli, et al., 2002.

En el lado derecho de la Figura 7, se representa el gráfico de vectores asociados a la corriente y voltaje; la relación entre el voltaje de la membrana, el voltaje de la fuente y el voltaje del circuito se puede describir con ayuda de las ecuaciones 11 a 13

$$
\begin{aligned}
& V_{1} \operatorname{Cos}\left(\phi-\phi_{1}\right)+V_{B L M} \operatorname{Cos}\left(\phi_{B L M}-\phi\right)=V_{S} \\
& V_{1} \operatorname{Sin}\left(\phi-\phi_{1}\right)=V_{B L M} \operatorname{Sin}\left(\phi_{B L M}-\phi\right) \\
& \mathrm{w}_{B L M} V_{B L M}=I \operatorname{Sinj}{ }_{B L M}
\end{aligned}
$$

La corriente I enunciada por la ecuación 14 es la suma vectorial de las corrientes que atraviesan la resistencia $\mathrm{R}_{1}$ y la capacitancia $\mathrm{C}_{1}$ del instrumento de medida:

$$
I=V_{1} \sqrt{\left(\omega C_{1}\right)^{2}+\left(\frac{1}{R_{1}}\right)}
$$


Si la capacitancia de la bicapa lipídica plana ya ha sido medida, así como los ángulos de fase $\varphi_{\text {вьм }}$ у $\varphi$, el voltaje $V_{\text {BLM }}$ puede ser determinado. La resistencia de la bicapa lipídica plana se obtiene a partir de la relación enunciada por la ecuación 15

$$
R=\frac{V_{B L M}}{I \operatorname{Cos} \phi}
$$

\section{CONCLUSIONES}

Se realizó una revisión sobre la estructuración del concepto de membrana, fundamentada en los trabajos desarrollados a lo largo de varios años y su aporte al concepto actual de bicapa lipídica plana y su consolidación como la mejor representación para el estudio y caracterización de las membranas biológicas.

Se analizaron las propiedades y características que llevaron a plantear el comportamiento eléctrico de la membrana como la de un circuito eléctrico en paralelo RC, donde la resistencia y la capacitancia cobran un papel importante ya que permiten caracterizar el comportamiento de las membranas al observar cambios en estos parámetros, cuando son sometidas a interacciones con agentes físicos o químicos externos.

Se enuncian las principales propiedades y características de los métodos y técnicas utilizados, en la cuantificación de las propiedades eléctricas de las membranas de bicapa lipídica plana.

La exploración de los parámetros eléctricos de las BLM goza de interés en el plano científico, ya que permite avanzar en la evaluación y desempeño de una diversidad de agentes fisicoquímicos de interés en el área médica y farmacológica. 


\section{REFERENCIAS BIBLIOGRÁFICAS}

Alberts, B., Bray, D., Johnson, A., Lewis, J., Ralf, M., Roberts, K. \& Walter, P. (1998). Essential Cell Biology. New York: Garland Publishing

Bell, D. J., Coster, H. G. L. \& Smith, J. R. (1975). A computer based, four terminal impedance measuring system for low frequencies (designed for plasma membrane of living cells). Journal of Physics $E$ : Scientific Instruments, 8(1), 66-70

Benz, R. \& Janko, K. (1976). Voltage induced capacitance relaxation of lipid bilayer membranes; effects on membrane composition. Biochimica et Biophysica Acta, 455(3), 721-738.

Cevc, G. \& Marsh, D. (1987). Phospholipid Bilayers. Physical Principles and Models. New York: John Wiley $\&$ Sons

Coster, H. G. L. \& Simons, R. (1970). Anomalous dielectric dispersion in bimolecular lipid membranes. Biochimica et Biophysica Acta (BBA)-Biomembranes, 203(1), 17-27

Coster, H. G. L. \& Smith. J. R. (1974). The molecular organization of bimolecular lipid membranes. A study of the low frequency Maxwell-Wagner impedance dispersion. Biochimica et Biophysica Acta (BBA) - Biomembranes, 373(2), 151-164

Coster, H. G. L. (1999). Self-assembly, stability and the electrical characteristics of cell membranes. Australian Journal of Physics, 52(1), 117-141

Danielle, J. F. \& Davson, H. A. (1935). A contribution to the theory of permeability of thin films. Journal of Cellular and Comparative Physiology. 5(4), 495-508

De Robertis, E. D .P., Nowinski, W. W. \& Caez, F. A. (1970). Cellular Biology. Philadelphia: W. B. Saunders Company

Diederich, A., Bahr, G. \& Winterhalter, M. (1998). Influence of surface charges on the rupture of black lipid membranes. Physical Review E, 58(4), 4883-4889

Fettiplace, R., Andrews, D. M. \& Haydon, D. A. (1971). The thickness, composition and structure of some lipid bilayers and natural membranes. Journal of Membrane Biology, 5(3), 277-296.

Finean, J. B., Coleman, R. \& Mitchell, R. H. (1978). Membranes and their Cellular Functions, 2nd ed. London: Blackwell Scientific Publications

Fricke, J. H. \& Morse, S. (1925). The electric capacity of suspensions with special reference to blood. Journal of General Physiology, 9(2), 137-152. 
Gallucci, E., Micelli, S. \& Monticelli, G. (1996). Pore formation in lipid bilayer membranes made of phosphatidylinositol and oxidized cholesterol followed by means of alternating current. Biophysical Journal, 71(2), 824-831.

Goldup, A., Ohki, S. \& Danielli, J. F. (1970). A quantitative description of membrane current and its application to conduction and excitation in nerve. Recent Progress in Surface science Vol 3. New York: Academic Press

Gorter, E. \& Grendel, F. (1925). On bimolecular layers of lipoids on the chromocytes of the blood. Journal of Experimental Medicine, 41(4), 439-443.

Hanai, T., Haydon, D. A. \& Taylor, J. (1964). An investigation by electrical methods of lecitin-inhydrocarbon films in aqueous solutions. Proceedings of the Royal Society of London. Series A: Mathematical and Physical Sciences, 281(1386), 377-39.1

Hianik, T. (2006). Structure and physical properties of biomembranes and model membranes. Acta Physica Slovaca, 56(6), 687-805

Hober, R. (1910). Eine Methode, die elektrische Leitfahigkeit im Innern von Zellen zu messen. Pflügers Archiv für die gesamte Physiologie des Menschen und der Tiere, 133, 237-259.

Huang, W. T. \& Levitt, D. G. (1977). Theoretical calculation of the dielectric constant of a bilayer membrane. Biophysical Journal, 17(2), 111-112.

Ivkov, V. G. \& Berestovsky, G. N. (1981). Dynamic Structure of Lipid Bilayer. Moscow: Nauka

Jain, M. K. (1988). Introduction to Biological Membranes. New York: John Wiley \& Sons.

Kalinowski, S. \& Figaszewski, Z. (1992). A new system for bilayer lipid membrane capacitance measurements: method, apparatus and applications, Biochimica et Biophysica Acta, 1112(1), 57-66

Kramar, P., Miklavcic, D. \& Macek L. A. (2007). Determination of the lipid bilayer breakdown voltage by means of a linear rising signal. Bioelectrochemistry, 70, 23-27

Kramar, P., Miklavcic, D. \& Macek-Lebar, A. (2009). A system for the determination of planar lipid membrane voltage and its applications. IEEE Transactions on Nanobioscience, 8(2), 132-138

Kramar, P., Miklavcic, D., Kotulska, M. \& Macek L. A. (2010). Voltage and Current-Clamp Methods for Determination of Planar Lipid Bilayer Properties. Advances in Planar Lipid Bilayer and Liposomas, 11(2), 29-69

Langmuir, I. (1933). Oil Lenses on Water and the Nature of Monomolecular Expanded Films. Journal of Chemical Physics, 1(11), 756-776 
Langmuir, I. \& Waugh, D. F. (1938). The adsorption of proteins at oil water interfaces and artificial protein-lipid membranes. Journal of General Physiology, 21(6), 745-755

Läuger, P., Lesslauer, W., Marti, E. \& Richter, J. (1967). Electrical properties of bimolecular phospholipid membranes. Biochimica et Biophysica Acta (BBA)-Biomembranes,135(1), 20-32.

Meier, W., Graff, A., Diederich, A. \& Winterhalter, M. (2000). Stabilization of planar lipid membranes: a stratified layer approach. Biochimica et Biophysica Acta, 2000(2), 4559-4562.

Micelli, S., Gallucci, E. \& Picciarelli, V. (2000). Studies of mitochondrial porin incorporation parameters and voltage-gated mechanism with different black lipid membranes. Bioelectrochemistry, 52(1), 63-75

Micelli, S., Gallucci, E., Meleleo, D., Stipani, V. \& Picciarelli, P. (2002). Mitochondrial porin incorporation into black lipid membranes:ionic and gating contribution to the total current. Bioelectrochemistry, 75, 97-106

Mueller, P., Rudin, D. O., Tien, H. I. \& Wescott, W. C. (1962). Reconstitution of cell membrane structure in vitro and its transformation into an excitable system. Nature, 194, 979-980.

Mueller, P., Rudin, D. O., Tien, H. I. \& Wescott, W. C.. \& Danielli, J. J. (1964). En K.C.A. PANKHURST and A.C. Riddeford (eds). Recent Progress in Surface science Vol I. New York: Academic Press

Nagle, J. F. \& Tristram-Nagle, S. (2000). Reviews on Biomembranes. Biochimica et Biophysica Acta, 1469(3), 159-195.

Neumcke, B. \& Läuger, P. (1969). Nonlinear Electrical Effects in Lipid Bilayer Membranes: II. Integration of the Generalized Nernst-Planck Equations. Biophysical Journal, 9(9), 1160-1170.

Overton, E. (1899). Ueber die allgemeinen osmotischen Eigenschaften der Zelle ihre vermutlichen Ursachen und ihre Bedevitung für die Physiologie. Vietljahrsschrift der Naturforschenden Gesellschaft in Zurich. 44, pp. 88-135.

Pum, D. \& Sleytr, U. B. (1999). The application of bacterial S-layers in molecular nanotechnology, TIBTECH, 17, 8-12

Robertson, J.D. (1959). The ultrastructure of cell membranes and their derivatives. Biochemical Society Symposium, 16, 3-43

Rogers, H. J., Perkins, H. R. \& Ward, J. B. (1980). Microbial Cell Wals and Membranes. London: Chapman and Hall

Roland, J. C., Szollosi, A. \& Szollosi, D. (1974) Atlas de Biologie Cellulaire, Paris: Masson et Cie 
Rosen, D. \& Sutton, A. M. (1968). The effects of a direct current potential bias on the electrical properties of bimolecular lipid membranes. Biochimica et Biophysica Acta, 163(2), 226-233

Schwan, H. P., Huang, C. \& Thompson, T. E. (1966) Proceedings Annual Meeting Biophysical Society 10 th, 51

Sharma, V., Uma-Maheswari, K., Murphy, J. C. \& Tung, L. (1996). Poloxamer 188 decreases susceptibility of artificial lipid membranes to electroporation. Biophysical Journal, 71(6), 3229-3241.

Sim, E. (1982). Membrane Biochemistry. New York: Chapman and Hall

Singer, S. J. \& Nicolson, G. L. (1972). The fluid mosaic model of the structure of cell membranes. Science, 175, 720-731.

Taylor, J. \& Haydon, D. A. (1966). Stabilization of thin films of liquid hydrocarbon by alkyl chain interaction. Discussions of the Faraday Society, 42, 51-59

Tien, H. T. (1966). Thickness and molecular organization of bimolecular lipid membranes in aqueous media. Journal of Molecular Biology, 16(2), 577-580

Tien, H. T., \& Dianna, A. L. (1967). Some physical properties of bimolecular lipid membranes produced from new lipid solutions. Nature, 215, 1199-1200.

Tien, H. T., Dianna, A. L. \& Louise, A. (1968). Bimolecular lipid membranes: a review and a summary of some recent studies. Chemistry and Physics of Lipids, 2(1), 55-101

Tien, H. T. (1974). Bilayer Lipid Membranes (BLM). Theory and Practice. New York: Marcell Dekker.

Tien, H. T. (1975). Thickness and molecular organization of bimolecular lipid membranes in aqueous media. Journal of Molecular Biology, 16(2), 577-580

Tien, H. T. \& Ottova, A. L. (2001). The lipid bilayer concept and its experimental realization: from soap bubbles, kitchen sink, to bilayer lipid membranes. Journal of Membrane Science, 189(1), 83-117

Troiano, G. C., Tung, L., Sharma, V. \& Stebe, K. J. (1998). The reduction in electroporation voltages by the addition of surfactant to planar lipid bilayer. Biophysical Journal, 75(2), 880-888

Vargas, J., Alarcón, J. M. \& Rojas, E. (2000). Displacement currents associated with the insertion of Alzheimer Disease amyloid (beta)-peptide into planar bilayer membranes. Biophysical Journal, 79(2), 934-944

White, S. H. (1970). A study of lipid bilayer membrane stability using precise measurements of specific capacitance. Biophysical Journal, 10(12), 1127-1147. 
Wilhelm, C., Winterhalter, M., Zimmermann, U. \& Benz, R. (1993). Kinetics o pore size during irreversible electrical breakdown of lipid bilayer membranes. Biophysical Journal, 64(1), 121-128.

Zetterquist, H. (1956). The Ultrastructural Organisation of the Columnar Epitelial Cells at Mouse Intestine. Thesis, from the Department of Anatomy, Karolinska Institute, Stockholm, Sweden. 